مقارنة بين المقدرات الاعتيادية والحصينة لنماذج السلاسل الزمنية المختلطة الثنائية من الرتب الانيا

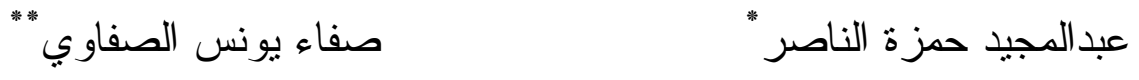

المستخلص

هنالك اهتمام متز ايد بالسلاسل الزمنية متعددة المتغيرات وخصوصا النماذج

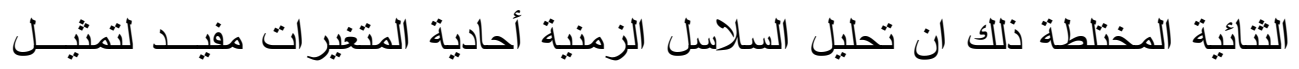
العلاقة لبيانات السلسلة الزمنية ، ولكن هذا التمثيل لا بأخذ بنظر الاعتبار العوامـلـل الاخرى او الظواهر التي تؤثز في الظاهرة بشكل اخــر • وهــــا التــر ابط بــين المتغير ات يمكن ان يوضـح من خلال در اسة السلاسل الزمنية ثنائيــة المتغيـر ات و التي تتضمن اكثر من ظاهرة لمدة من الزمن • ان الحصول على مقدر ات ذات كفاءة عالية يعد من اهم مر احـلـل التحليـلـل

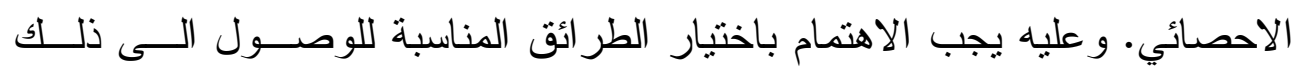
المستوى من التقدير وخصوصـا عندما تكون بيانات السلسلة تحتوي علــى بعــ المشاهدات الملوثة التي تكون غير منسقة مع بقية مشاهدات العينة. وهذا هو هدف البحث وهو اجر اء مقارنة بين مقدرات الامكان الاعظم و المقدرات الحصينة لتقدير معلمات النموذج المختلط ثنائي المتغير ات BARMA(1,1) وقد تبين من خــلال الدر اسة ان مقدرات الامكان الاعظم هي الافضل في حالة عدم اقحام الثوارد وفي حالة اقحام الثوارد بنسبة 10\% كانت المقدرات الحصينة هي الافضل وفي جميع الطر ائق تم استخدام منوسط الاخطاء النسبية المطلقة MAPE معيــار اً احصــائياً للمقارنة.

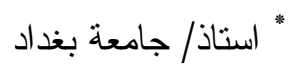

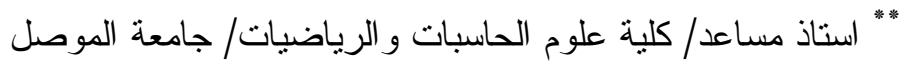
2005/8/14 : تاريخ القبول تاريخ التسلم : 9 /5 /2005 


\section{Comparison Between Ordinary Method and Robust Method to estimate the Parameters of the Bivariate Mixed Model, $\operatorname{ARMA}(1,1)$}

\section{ABSTRACT}

A condensed study was done to compare between the ordinary estimators. In particular the maximum likelihood estimator and the robust estimator, to estimate the parameters of the bivariate mixed model of order one, namely BARMA $(1,1)$.

Simulation experiments were done for varieties of BARMA(1,1), using small, moderate and large sample sizes, where some new results were obtained. MAPE was used as a statistical criterion for comparison.

$$
\text { المبحث الاول: المقدمة }
$$

من اكثر الطر ائق المستخدمة في تقدير معلمات نماذج السلاسل الزمنية هـي طريقة المربعات الصغرى Least Square Method التي يرمـز لهــا بــالرمز

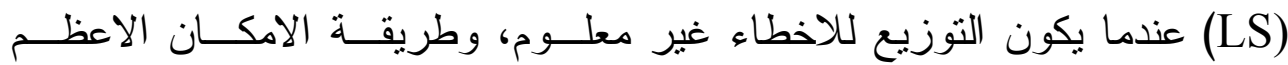
Maximum Likelihood Method

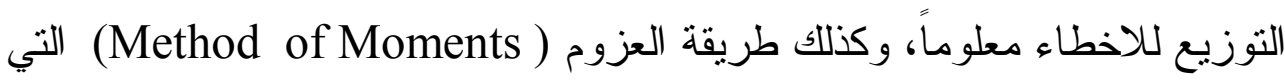

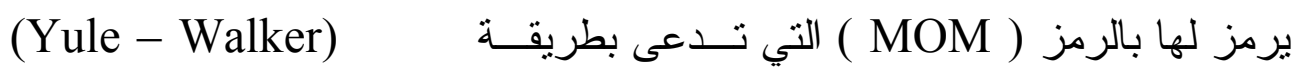
ايضاً .

ان مقدر ات هذه الطر ائق قد تكون كفو عة و منسقة ومناسبة في حالة تـــو افر

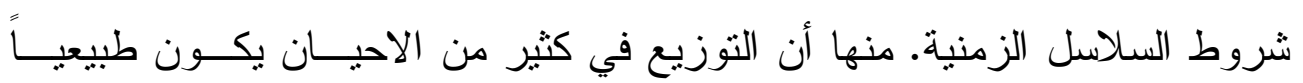

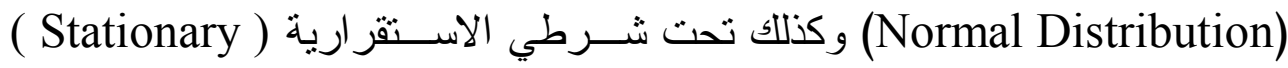
و الانعكاسية ( Invertibility)، ولكن عند اختلاف الثروط نتيجة لوجود عامـلـ

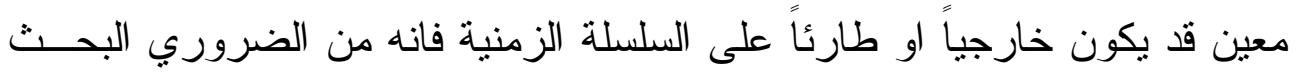

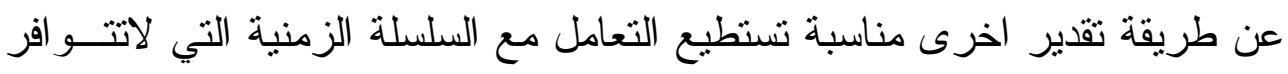

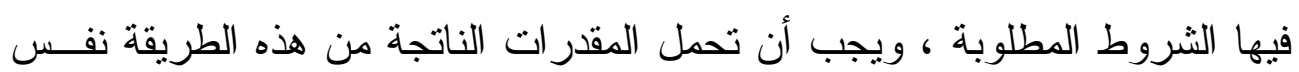

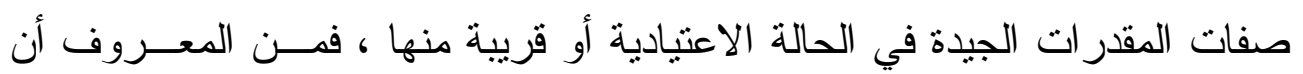

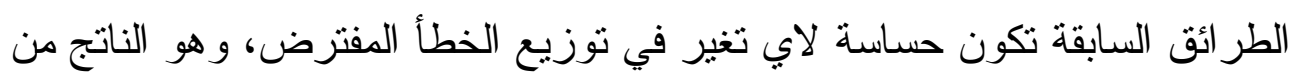


الاختلاف في نسق البيانات حتى وان كان صغير ا ، ان هذا التغير عادة ما يكــون

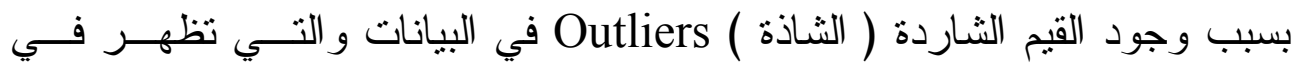

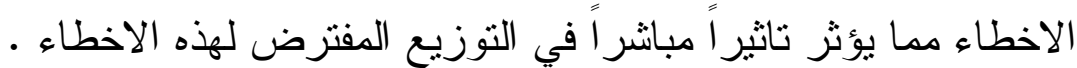

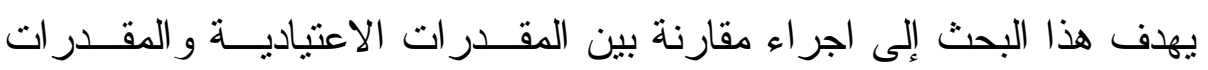

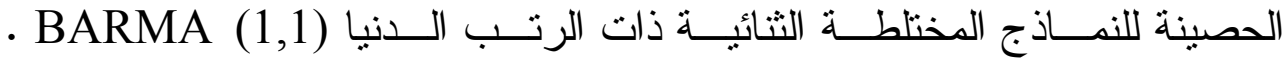
مستخدمين الاسلوب النظري ( النظرية الاحصائية ) و الاسلوب التجريبي (المحاكاة) لتقدير معلمات السلاسل الزمنية المولدة عشوائيا في حالة عدم تو افر الثوارد ومن الثن ثم في حالة اقحام الثو ارد و المقارنة بينهما باستخدام معيار متوسط الاخطاء النسبية المطلقة Mean Absolute Percentage Error) MAPE). ولقد تم استخدام هذا المعيار لكونه من المعايير الاكثر دقــة للمقارنـــة بــين طر ائق التقدير في السلاسل الزمنية، ولم يتم استخدام متوسط مربعسـات الاخطــاء لهاء (Mean Square Error) MSE

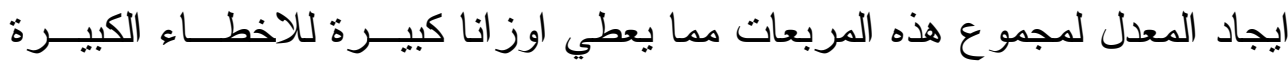

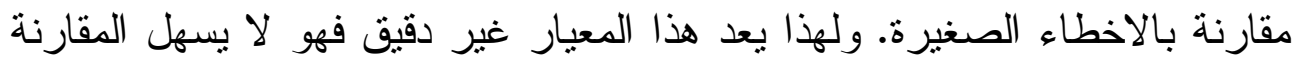

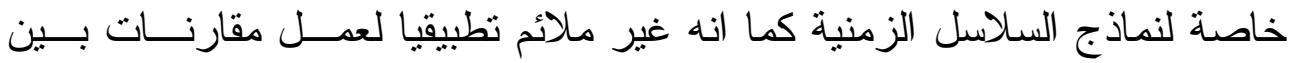
الطر ائق المختلفة للتقدير ( دانيال، 2004).

نموذج الاحدار الذاتي-الاوساط المتحركة المختلطة الثنائية من الرتبة الاولى BARMA(1,1) Models

نموذج BARMA (1,1) يكون على وفق الصيغة الاتية :

$$
\left(\mathrm{I}-\Phi_{1} \mathrm{~B}\right) \mathrm{y}_{\mathrm{t}}=\left(\mathrm{I}-\Theta_{1} \mathrm{~B}\right) \mathrm{a}_{\mathrm{t}}
$$

اولاًا : إن النموذج مستقر إذا كانت جذور المعادلة Iالوحدة Unit Circle أو إذا كانت القيم المميزة في 1 ه داخل دائرة الوحدة .

ثانياً : يمكن كتابة النموذج بدلالة الاخطاء العشو ائية وحسب الصيغة الاتية: 


$$
\mathrm{y}_{\mathrm{t}}=\sum_{\mathrm{s}=0}^{\infty} \Psi_{\mathrm{s}} \mathrm{a}_{\mathrm{t}-\mathrm{s}}
$$

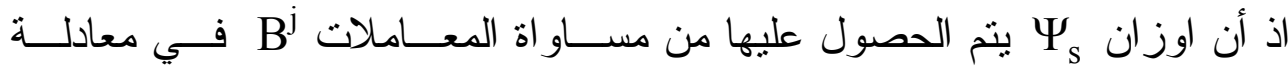

$$
\begin{aligned}
& \left(I-\Phi_{1} B\right)\left(I+\Psi_{1} B+\Psi_{2} B^{2}+\ldots .\right)=\left(I-\Theta_{1} B\right) \\
& \Psi_{j}=\Phi_{1} \Psi_{j-1}=\Phi_{1}^{j-1}\left(\Phi_{1}-\Theta_{1}\right) \quad, j \geq 1 \ldots \ldots .
\end{aligned}
$$

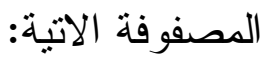

ويكون النموذج قابلا للعكس إذا كانت جذور المعادلة

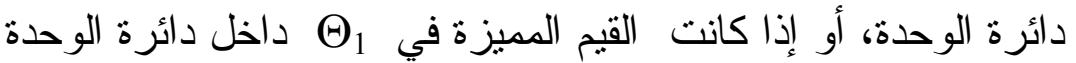

ثالثاً: كما ويمكن الثتقاق مصفوفة التباين المشترك على النحو الاتي: $\mathrm{E}\left[\mathrm{y}_{\mathrm{t}}\left(\mathrm{y}_{\mathrm{t}}-\mathrm{y}_{\mathrm{t}-1}^{\prime} \Phi^{\prime}{ }_{1}\right)\right]=\mathrm{E}\left[\left(\mathrm{y}_{\mathrm{t}-\mathrm{k}}\left(\mathrm{a}_{\mathrm{t}}{ }_{\mathrm{t}}-\mathrm{a}_{\mathrm{t}-1}{ }^{\prime} \Theta_{1}{ }_{1}\right)\right]\right.$

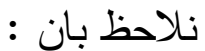

$\mathrm{E}\left[\mathrm{y}_{\mathrm{t}}\left(\mathrm{a}_{\mathrm{t}-1} \theta_{1}\right)\right]=\mathrm{E}\left[\left(\Phi_{1} \mathrm{y}_{\mathrm{t}-1}+\mathrm{a}_{\mathrm{t}}-\Theta_{1} \mathrm{a}_{\mathrm{t}-1}\right)\left(\mathrm{a}_{\mathrm{t}-1}^{\prime} \Theta_{1}^{\prime}\right)\right]$ $=\Phi_{1} \sum \Theta^{\prime}{ }_{1}-\Theta_{1} \sum \Theta_{1}^{\prime}$

$\Gamma(0)-\Gamma^{\prime}(1) \sum=\sum-\left(\Phi_{1}-\Theta_{1}\right) \sum \Theta_{1} \quad$ ، $\mathrm{k}=0$

$\Gamma(1)-\Gamma(0) \Phi_{1}^{\prime}=-\sum \Theta_{1}^{\prime} \quad$ ، $\quad \mathrm{k}=1$

$\Gamma(\mathrm{k})-\Gamma^{\prime}(\mathrm{k}-1) \Phi^{\prime}{ }_{1}=0 \quad$ ، $\mathrm{k} \geq 2$

$$
\Gamma(\mathrm{k})= \begin{cases}\Gamma(1) \sum+\sum-(\Phi-\Theta) \sum \Theta & , \mathrm{k}=0 \\ \Gamma(0) \Phi_{1}^{\prime}-\sum \Theta_{1} & , \mathrm{k}=1 \\ \Gamma(\mathrm{k}-1) \Phi_{1}^{\prime} & , \mathrm{k} \geq 2\end{cases}
$$

$$
\begin{gathered}
\text { النموذج المختلط ثنائي المتغيرات } \mathbf{B A R M A ( 1 , 1 )} \\
\left(\mathrm{I}-\Phi_{1} \mathrm{~B}\right) \mathrm{y}_{\mathrm{t}}=\left(\mathrm{I}-\Theta_{1} \mathrm{~B}\right) \varepsilon_{\mathrm{t}} \\
\Phi_{1}=\left[\begin{array}{ll}
\phi_{11} & \phi_{12} \\
\phi_{21} & \phi_{22}
\end{array}\right] \quad \text { ، } \Theta_{1}=\left[\begin{array}{ll}
\theta_{11} & \theta_{12} \\
\theta_{21} & \theta_{22}
\end{array}\right]
\end{gathered}
$$




$$
\begin{gathered}
\text { و ان مصفوفة التباين و التباين المشترك للموجه }\left[\begin{array}{l}
\varepsilon_{1 t} \\
\varepsilon_{2 t}
\end{array}\right] \text { (Vector) } \\
\sum=\left[\begin{array}{ll}
\sigma_{11} & \sigma_{12} \\
\sigma_{21} & \sigma_{22}
\end{array}\right]
\end{gathered}
$$

و على فرض ان توزيع الموجه $]$ ان

فمن الو اضح ان لوغاريتم دالة الامكـــان (Bivariate Normal Distribution)

للعينة يمكن كتابته على وفق الصيغة الاتية:

$$
\ell \mathrm{nL}\left(\Phi_{1}, \theta_{1}, \sum \mid \mathrm{y}\right)=\mathrm{cons} \tan \mathrm{t}-\frac{\mathrm{n}}{2} \ell \mathrm{n}\left|\sum\right|-\frac{1}{2} \sum_{\mathrm{t}=1}^{\mathrm{n}} \varepsilon_{\mathrm{t}}^{\mathrm{T}} \sum^{-1} \varepsilon_{\mathrm{t}}
$$

و التي يمكن كتابتها على وفق الصيغة الاتية :

$$
\ell \mathrm{nL}=\text { cons tan } \mathrm{t}-\frac{\mathrm{n}}{2} \ell \mathrm{n}\left|\sum\right|-\frac{1}{2} \operatorname{tr} \sum^{-1} \mathrm{~S}\left(\Phi_{1}, \Theta_{1}\right) \ldots \ldots
$$

$$
\mathrm{S}\left(\Phi_{1}, \Theta_{1}\right)=\sum_{\mathrm{t}=1}^{\mathrm{n}} \varepsilon_{\mathrm{t}} \varepsilon_{\mathrm{t}}^{\mathrm{T}}
$$

حيث ان

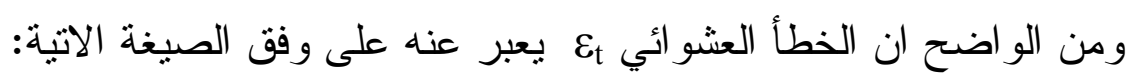

$$
\varepsilon_{\mathrm{t}}=\mathrm{y}_{\mathrm{t}}-\Phi_{1} \mathrm{y}_{\mathrm{t}-1}+\Theta_{1} \varepsilon_{\mathrm{t}-1}
$$

هكذا فان مقدر ات الامكان الاعظم لمصفوفات المعلمات

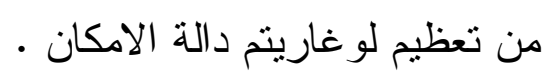
ويعتبر نموذج الانحدار الذاتي ثنائي المتغيرات

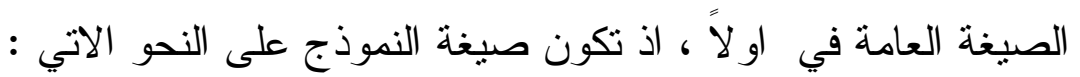

$$
\left[\begin{array}{l}
\mathrm{y}_{1, \mathrm{t}} \\
\mathrm{y}_{2, \mathrm{t}}
\end{array}\right]=\left[\begin{array}{ll}
\phi_{11} & \phi_{12} \\
\phi_{21} & \phi_{22}
\end{array}\right]\left[\begin{array}{c}
\mathrm{y}_{1, \mathrm{t}-1} \\
\mathrm{y}_{2, \mathrm{t}-1}
\end{array}\right]+\left[\begin{array}{c}
\varepsilon_{1, \mathrm{t}} \\
\varepsilon_{2, \mathrm{t}}
\end{array}\right] \ldots
$$

ويصبح لو غاريتم دالة الامكان للعينة بالصيغة الاتية :

$$
\ell \mathrm{nL}\left(\Phi_{1}, \sum \mid \mathrm{y}\right)=\mathrm{cons} \tan \mathrm{t}-\frac{\mathrm{n}}{2}\left|\sum\right|-\frac{1}{2} \sum_{\mathrm{t}=1}^{\mathrm{n}} \varepsilon_{\mathrm{t}}^{\mathrm{T}} \sum^{-1} \varepsilon_{\mathrm{t}}
$$


وباستخدام خصائص المصفوفات يمكن كتابــة لوغــاريتم دالــة الامكـــان للعينــة على وفق الصيغة الاتية: $\left(\mathrm{y}_{1}, \mathrm{y}_{2}, \ldots, \mathrm{y}_{\mathrm{n}}\right)$

$$
\ell \mathrm{nL}=\mathrm{cons} \tan \mathrm{t}-\frac{\mathrm{n}}{2}\left|\sum\right|-\frac{1}{2} \operatorname{tr} \sum^{-1} \mathrm{~S}\left(\Phi_{1}\right)
$$

$$
S\left(\Phi_{1}\right)=\sum_{t=1}^{n} \varepsilon_{t} \varepsilon_{t}^{T}
$$

ويعبر عن حد الخطأ العثوائي بالصيغة الاتية:

$$
\varepsilon_{\mathrm{t}}=\mathrm{y}_{\mathrm{t}}-\Phi_{1} \mathrm{y}_{\mathrm{t}-1}
$$

كما ان النموذج المختلط ثنائي المتغير ات BARMA(0,1) هو الاخر حالة خاصة من الحالة العامة في اولاً ، اذ يمكن كتابة النموذج على وفق الصيغة الاتية:

$$
\left[\begin{array}{l}
\mathrm{y}_{1, \mathrm{t}} \\
\mathrm{y}_{2, \mathrm{t}}
\end{array}\right]=\left[\begin{array}{ll}
1 & 0 \\
0 & 1
\end{array}\right]\left[\begin{array}{l}
\varepsilon_{1, \mathrm{t}} \\
\varepsilon_{2, \mathrm{t}}
\end{array}\right]-\left[\begin{array}{ll}
\theta_{11} & \theta_{12} \\
\theta_{21} & \theta_{22}
\end{array}\right]\left[\begin{array}{c}
\varepsilon_{1, \mathrm{t}-1} \\
\varepsilon_{2, \mathrm{t}-1}
\end{array}\right] \ldots \ldots \ldots
$$

ويمكن بعد التبسيط كتابة لو غاريتم دالة الامكان لعينة حجمها n بالصيغة الاتية:

$$
\ell \mathrm{nL}=\text { cons tan } \mathrm{t}-\frac{\mathrm{n}}{2} \ell \mathrm{n}\left|\sum\right|-\frac{1}{2} \operatorname{tr} \sum^{-1} \mathrm{~S}\left(\theta_{1}\right)
$$

$$
S\left(\theta_{1}\right)=\sum_{t=2}^{n} \varepsilon_{t} \varepsilon_{t}^{1}
$$

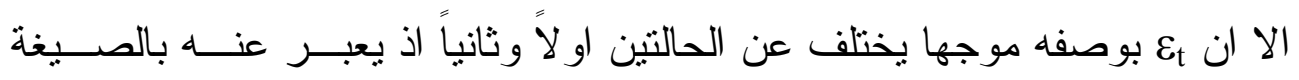
الاتية:

$$
\varepsilon_{\mathrm{t}}=\mathrm{y}_{\mathrm{t}}+\Theta_{1} \varepsilon_{\mathrm{t}-1}
$$

\section{تقدير معلمات النموذج بالطريقة الحصينة :}

إن القيم الثاردة للسلسلة الزمنية يمكن أن تؤثر عكسيا في كل من مقدرات المربعات الصغرى (LS) ومعامل مقدر ات M لمعلمــات الأنحــدار الــذاتي • إن الن الأهتمام إنصب هنا للحصول على مقدر ات حصينة لمعامل الأنحدار الــذاتي مـنـ

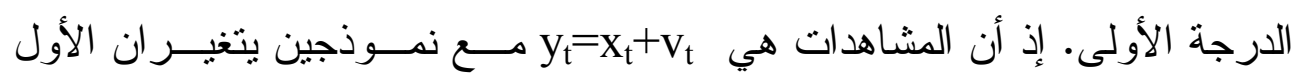

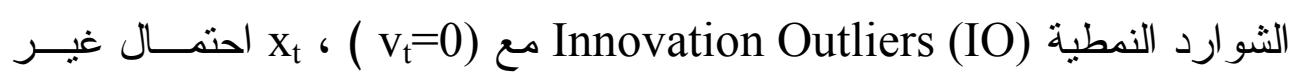
طبيعي Possibly non-Gaussian و الثاني نموذج الثوارد المضافة Pdditive 


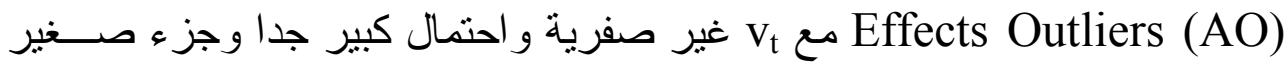
W من الزمن و $X_{t}$ possibly quite large small fraction

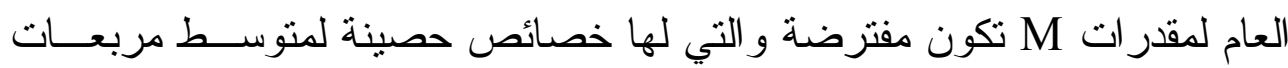

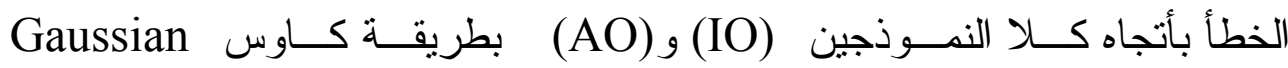
.Method

في هذا المبحث سندرس مشاكل الحصول على مقدر ات حصــينة للأنحـــار

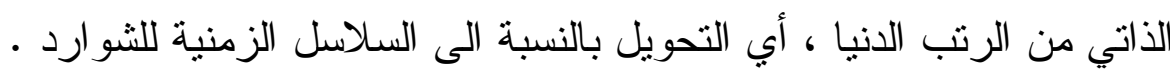

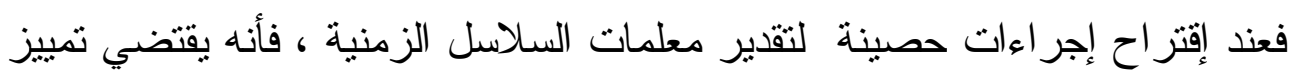

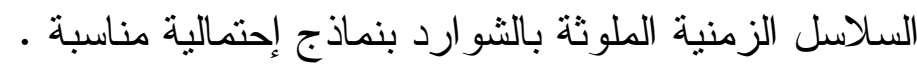

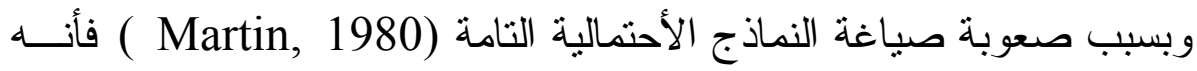

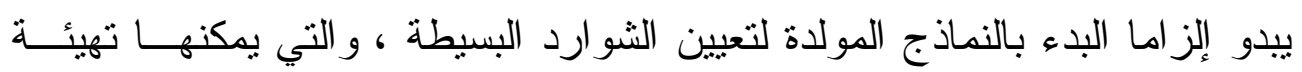

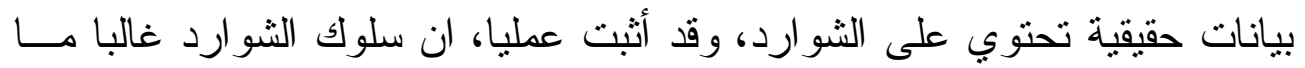

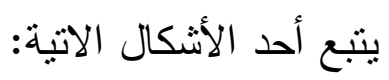

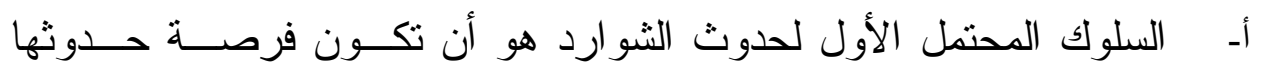

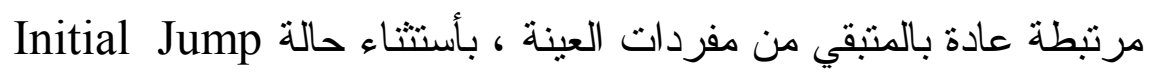
التي تعرف بالتغير الفجائي الأبندائي .

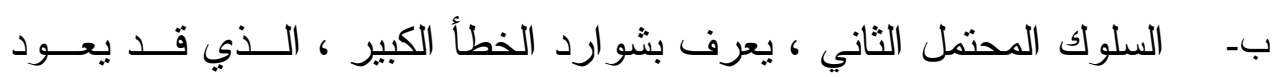

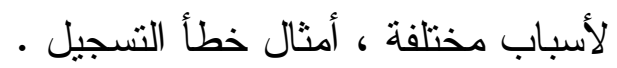

ج- السلوك المحتمل الثالث ، يعرف بالثوال ارد مختلفة الأنواع ذات السلوك اللا

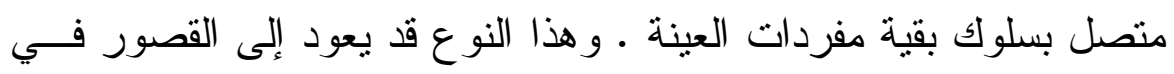
إستخدام وسيلة التسجيل.

مما تقدم ، فأن أنواع السلوك المحتملة أعلاه يمكن أن تتحقق بنماذج مناسبة ،

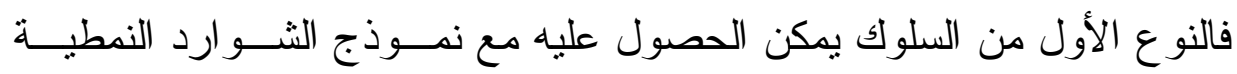

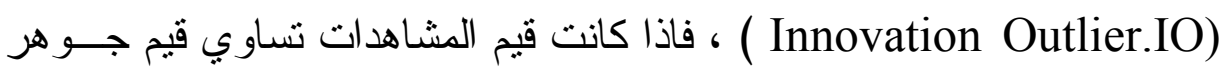
العملية (Zch , 1979)، أي (13) (13)

$\mathrm{y}_{\mathrm{i}}=\hat{x_{t}}$ 
و إن توزيع أخطاء (IO) متتاظر ، نقيل الأطر اف ، عندئذ فأن نموذج الثــــوارد

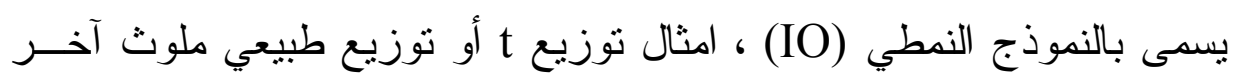

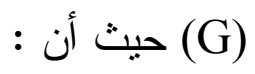

$$
\begin{aligned}
& \mathrm{G}\left(\mathrm{p}, \sigma_{1}, \sigma_{2}\right)=(1-\mathrm{p}) \mathrm{N}\left(0, \sigma^{2}\right)+\mathrm{pN}\left(\left(0, \sigma^{2}{ }_{2}\right)\right. \\
& \text { بحيث ان }
\end{aligned}
$$

وبعبارة أخرى إذا كانت قيم ( Et ) الضوضاء الأبيض تحقق شرط ( iid ) للمتغير

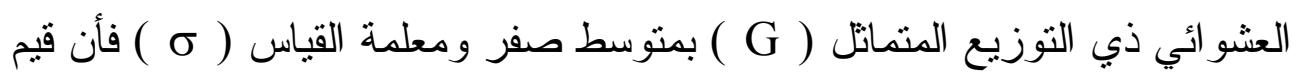

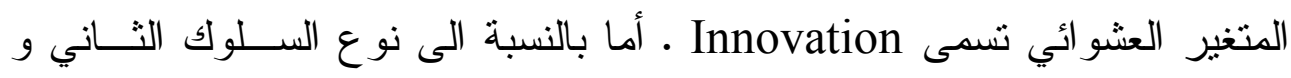

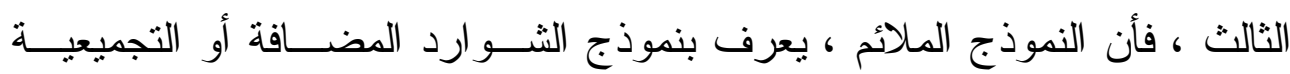
: Additive outlier (AO)

$$
\mathrm{Y}_{\mathrm{t}}=\mathrm{X}_{\mathrm{t}}+\mathrm{V}_{\mathrm{t}}
$$

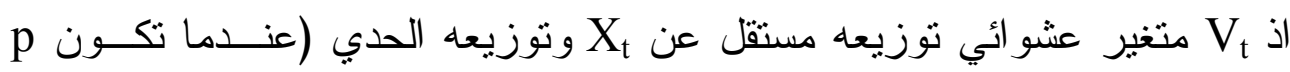
صغيرة نسبيا) هو

$$
\mathrm{P}\left(\mathrm{V}_{\mathrm{t}}=0\right)=1-\gamma
$$

هذا وقد اثتتت التجربة في حقل السلاسل الزمنية، ان مدى ل ع يتحقق ما بين (Stochinger and Duter, 1987) (0.25,0.01) توزيع V طبيعيا مختلطا

$$
\operatorname{CND}\left(p, \sigma_{3}\right)=(1-\gamma) \delta_{0}+\gamma \mathrm{N}\left(\mathrm{p}, \sigma_{3}^{2}\right)
$$

اذ تثير $\delta_{0}$ الثقل

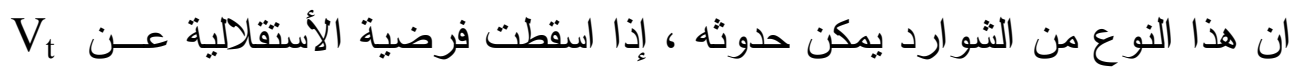

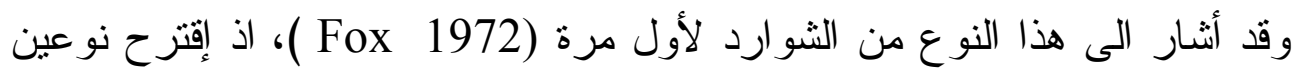

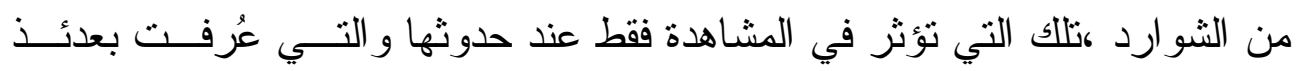

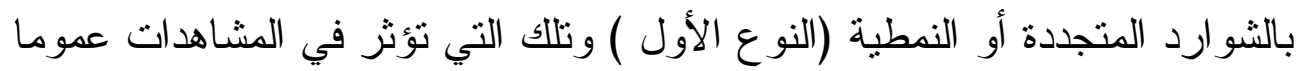

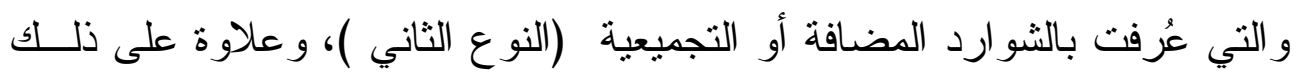


فقد تقدم Fox في السنة نفسها أيضا بإقتر احين لتحديد نوع الثوارد ، الأول يبنـى على فكرة الفحص للنموذجين ومن ثم إختيار النموذج الذي تكون المشاهدة الثشاردة

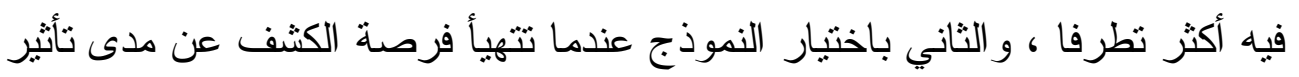

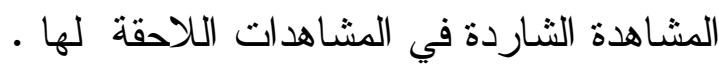
الجانب التجريبي

\section{صياغة نموذج المحاكاة :}

تعد المحاكاة عملية تتبيه او تقلبد للو اقع الحقيقي ، أي ايجاد صورة طبق الاصــل

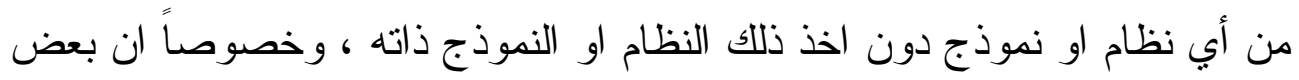

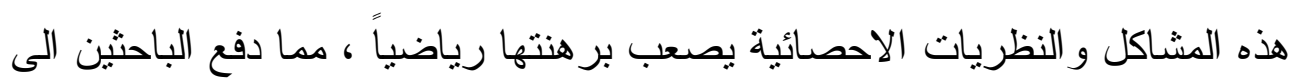

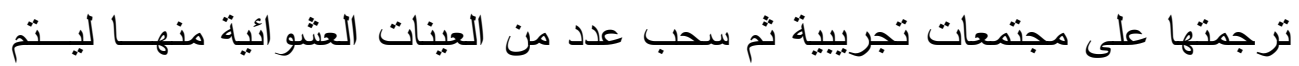

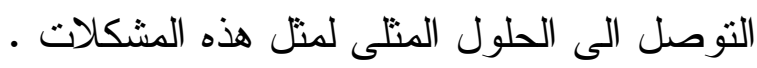

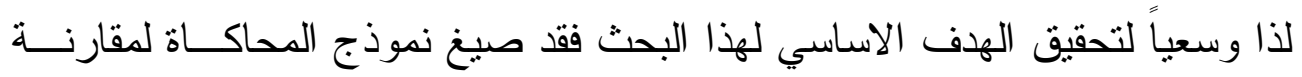

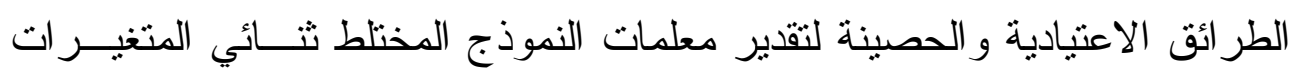
BARMA $(1,1)$

أ : مرحلة توليد البيانات لغرض تقدير معلمات النموذج (1,1) BARMA $\Phi(\mathrm{B}) \mathrm{y}_{\mathrm{t}}=\Theta(\mathrm{B}) \varepsilon_{\mathrm{t}}$ $\varepsilon_{\mathrm{T}}=\Theta^{-1}(\mathrm{~B}) \phi(\mathrm{B}) \mathrm{y}_{\mathrm{t}}$ $\varepsilon_{\mathrm{t}}^{\prime} \varepsilon_{\mathrm{t}}=\mathrm{y}_{\mathrm{t}}^{\prime} \phi^{\prime}(\mathrm{B}) \theta^{-1}(\mathrm{~B}) \theta^{-1}(\mathrm{~B}) \phi(\mathrm{B}) \mathrm{y}_{\mathrm{t}}$ اذ ان من المعادلة الاخيرة تم اثنقاق ( (الذ اذ ان الموجه $\varepsilon_{\mathrm{t}} \sim \mathrm{N}_{2}\left[\left(\begin{array}{l}0 \\ 0\end{array}\right),\left(\begin{array}{ll}1 & 0 \\ 0 & 1\end{array}\right)\right]$

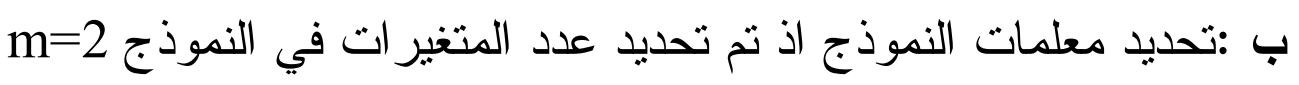
جـ : تحديد معلمات افتز اضية تحقق الاستقر ارية وقابلية العكس وحسب التشكيلة (التتويعة) الاتية: 


$$
\begin{aligned}
& \Phi=\left[\begin{array}{cc}
0.8 & -0.2 \\
0.2 & -0.6
\end{array}\right] \ldots ., \quad, \ldots . \Theta=\left[\begin{array}{cc}
0.6 & 0.4 \\
0.5 & -0.6
\end{array}\right] \\
& \Phi=\left[\begin{array}{cc}
0.8 & 0.1 \\
0.1 & 0.2
\end{array}\right] \ldots,, \ldots . \Theta=\left[\begin{array}{cc}
-0.6 & 0.3 \\
-0.3 & -0.8
\end{array}\right] \\
& \Phi=\left[\begin{array}{cc}
0.8 & -0.1 \\
-0.2 & 0.2
\end{array}\right] \ldots,, \ldots . \Theta=\left[\begin{array}{cc}
-0.2 & 0.6 \\
-0.6 & 0.2
\end{array}\right] \\
& \Phi=\left[\begin{array}{cc}
0.8 & -0.6 \\
0.5 & 0.6
\end{array}\right] \ldots ., \ldots . \Theta=\left[\begin{array}{cc}
-0.2 & 0.1 \\
-0.5 & 0.2
\end{array}\right] \\
& \Phi=\left[\begin{array}{cc}
0.8 & -0.6 \\
0.2 & 0.8
\end{array}\right] \ldots,, \ldots \Theta=\left[\begin{array}{cc}
-0.8 & 0.4 \\
0.3 & 0.8
\end{array}\right] \\
& \Phi=\left[\begin{array}{cc}
0.8 & 0.2 \\
-0.2 & 0.6
\end{array}\right] \ldots,, . . \Theta=\left[\begin{array}{cc}
-0.6 & 0.2 \\
0.2 & 0.6
\end{array}\right] \\
& \Phi=\left[\begin{array}{ll}
0.6 & 0.4 \\
0.3 & 0.2
\end{array}\right] \ldots,, \ldots \Theta=\left[\begin{array}{ll}
0.3 & 0.1 \\
0.2 & 0.5
\end{array}\right] \\
& \Phi=\left[\begin{array}{ll}
0.9 & 0.1 \\
0.2 & 0.7
\end{array}\right] \ldots,, \ldots \Theta=\left[\begin{array}{ll}
0.5 & 0.2 \\
0.2 & 0.7
\end{array}\right] \\
& \Phi=\left[\begin{array}{cc}
0.6 & -0.4 \\
-0.3 & 0.2
\end{array}\right] \ldots,, \ldots . \Theta=\left[\begin{array}{cc}
-0.3 & 0.1 \\
0.2 & 0.5
\end{array}\right] \\
& \Phi=\left[\begin{array}{cc}
-0.8 & -0.6 \\
-0.5 & 0.4
\end{array}\right] \ldots,, \ldots . \Theta=\left[\begin{array}{cc}
-0.2 & 0.1 \\
-0.5 & 0.3
\end{array}\right] \\
& \Phi=\left[\begin{array}{cc}
-0.8 & 0.6 \\
0.6 & -0.8
\end{array}\right] \ldots \ldots, \quad, \ldots \Theta=\left[\begin{array}{cc}
-0.8 & 0.6 \\
0.6 & -0.8
\end{array}\right] \\
& \Phi=\left[\begin{array}{cc}
-0.8 & 0.2 \\
0.2 & -0.8
\end{array}\right] \ldots,, \ldots \Theta=\left[\begin{array}{cc}
-0.8 & 0.2 \\
0.2 & -0.8
\end{array}\right] \\
& \Phi=\left[\begin{array}{cc}
-0.9 & 0.1 \\
0.2 & 0.7
\end{array}\right] \ldots,, \ldots . \Theta=\left[\begin{array}{cc}
0.8 & 0.4 \\
0.6 & -0.5
\end{array}\right]
\end{aligned}
$$

د : تحديد حجم العينة (n) اذ نم تحديد ثلاثة احجام للعينات هي ( 25 , 50 , 100 ) وتم تقدير معلمات النموذج المختلط ثنائي المتغيرات على وفق الطريقتين الاعتيادية و الحصينة ونم اقحام الثوارد بنسة 10\% وتم إعتماد القيمة المطلقة لمتوسط

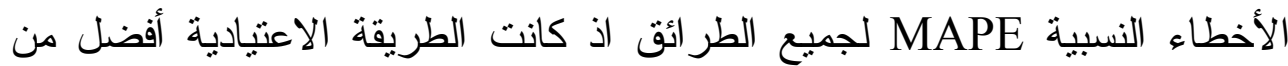
الطريقة الحصينة قبل إقحام الثوارد ، أما في حالة إقحام الثوارد بنسبة 10\% 
فكانت الطريقة الحصينة هي الأفضل وكما موضح في الجدول (1) بالنسبة للعينة

$$
\text { بحجم(25). (انظر (الصفاوي، 2005) بالنسبة الى العينات } 50 \text { و 100) . }
$$

\section{الاستتناجات}

i. في حالة كون حجم العينة صغير اً فان مقدر الامكان الاعظــم باعتمــاد معيـار متوسط الاخطاء النسبية (MAPE) هو المقدر المفضل بنسبة 87.5\% في حالة عدم اقحام الثوارد في حين تفوقت الطريقة الحصينة عند وجود الثوارد وبنسبة

$$
.95 \%
$$

ii في حالة كون حجم العينة متوسطاً فان مقدر الامكان الاعظــم باعتمــاد معيـار متوسط الاخطاء النسبية (MAPE) هو المقدر المفضل بنسبة 95\% في حالـــة عدم اقحام الثوارد في حين تفوقت الطريقة الحصينة عند وجود الثوارد وبنسبة

$$
.93 \%
$$

1ii متوسط الاخطاء النسبية (MAPE) هو المقدر المفضل بنسبة 97\% في حالـــة عدم تو افر الثوارد في حين تفوقت الطريقة الحصينة عند وجود الثوارد وبنسبة $.97 \%$ 


\section{(1) (الجدول (1) (2)}

يبين قيم متوسط الاخطاء النسبية المطلة MAPE لمعلمات نموذج BARMA(1,1 المقدرة عندما يكون حجم العينة (25)

\begin{tabular}{|c|c|c|c|c|c|c|c|}
\hline \multicolumn{5}{|c|}{ قبل التلويث } & \multicolumn{3}{|c|}{ بعد التلويث } \\
\hline رقم التشكيلة & طريقة التقديري القيم اضية للمعلمات & ML & Robust & Best & ML & Robust & Best \\
\hline 1 & $\begin{array}{l}\phi_{11}=0.8 \\
\phi_{12}=-0.2 \\
\phi_{21}=0.2 \\
\phi_{22}=-0.6 \\
\theta_{11}=0.6 \\
\theta_{12}=0.4 \\
\theta_{21}=0.5 \\
\theta_{22}=-0.6\end{array}$ & $\begin{array}{l}0.00191551 \\
0.00245530 \\
0.00165431 \\
0.00221439 \\
0.00174262 \\
0.00276808 \\
0.00237627 \\
0.00218199\end{array}$ & $\begin{array}{l}0.0794192 \\
0.0020000 \\
0.0020000 \\
0.0020000 \\
7.7386600 \\
1.8020000 \\
1.4420000 \\
1.5308200 \\
\end{array}$ & $\begin{array}{l}\text { ML } \\
\text { RO } \\
\text { ML } \\
\text { RO } \\
\text { ML } \\
\text { ML } \\
\text { ML } \\
\text { ML }\end{array}$ & $\begin{array}{l}0.00050000 \\
0.00200000 \\
0.00200000 \\
0.00533333 \\
0.00173600 \\
0.00269300 \\
0.00231680 \\
0.00246200\end{array}$ & $\begin{array}{l}0.000306387 \\
0.000000200 \\
0.000000200 \\
0.000000200 \\
0.000773866 \\
0.000180200 \\
0.000144200 \\
0.000153082\end{array}$ & $\begin{array}{l}\text { RO } \\
\text { RO } \\
\text { RO } \\
\text { RO } \\
\text { RO } \\
\text { RO } \\
\text { RO } \\
\text { RO }\end{array}$ \\
\hline 2 & $\begin{array}{l}\phi_{11}=0.8 \\
\phi_{12}=0.1 \\
\phi_{21}=0.1 \\
\phi_{22}=0.2 \\
\theta_{11}=-0.6 \\
\theta_{12}=0.3 \\
\theta_{21}=-0.3 \\
\theta_{22}=-0.8\end{array}$ & $\begin{array}{l}0.001942910 \\
0.002479630 \\
0.001736420 \\
0.002167800 \\
0.001761250 \\
0.002716140 \\
0.002328190 \\
0.002132190\end{array}$ & $\begin{array}{c}0.0816621 \\
0.0020000 \\
0.0020000 \\
0.0020000 \\
98.3582000 \\
1.8020000 \\
1.8420000 \\
0.3077610\end{array}$ & $\begin{array}{l}\text { ML } \\
\text { RO } \\
\text { RO } \\
\text { RO } \\
\text { ML } \\
\text { ML } \\
\text { ML } \\
\text { ML }\end{array}$ & $\begin{array}{l}0.0005000 \\
0.0020000 \\
0.0020000 \\
0.0053333 \\
0.0017360 \\
0.0026930 \\
0.0023168 \\
0.0024620\end{array}$ & $\begin{array}{c}0.00027991 \\
0.00000020 \\
0.00000020 \\
0.00000020 \\
0.00974395 \\
0.00018770 \\
0.00015020 \\
0.000033175\end{array}$ & $\begin{array}{l}\text { RO } \\
\text { RO } \\
\text { RO } \\
\text { RO } \\
\text { ML } \\
\text { RO } \\
\text { RO } \\
\text { RO }\end{array}$ \\
\hline
\end{tabular}




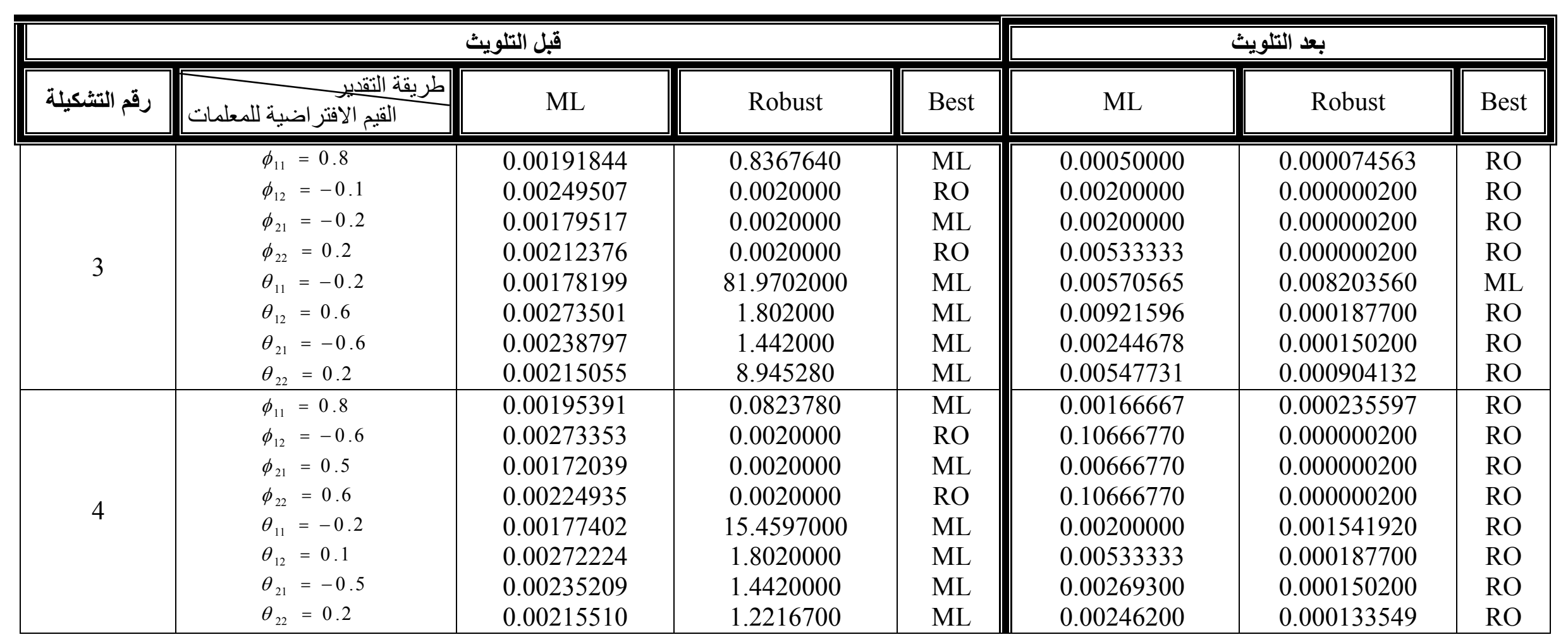




\begin{tabular}{|c|c|c|c|c|c|c|c|}
\hline \multicolumn{5}{|c|}{ قبل التلويث } & \multicolumn{3}{|c|}{ بعد التلويث } \\
\hline رقم التشكيلة & 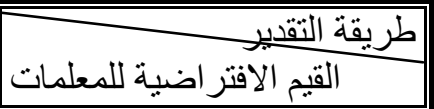 & ML & Robust & Best & ML & Robust & Best \\
\hline 5 & $\begin{array}{l}\phi_{11}=0.8 \\
\phi_{12}=-0.6 \\
\phi_{21}=0.2 \\
\phi_{22}=-0.8 \\
\theta_{11}=-0.8 \\
\theta_{12}=0.4 \\
\theta_{21}=0.3 \\
\theta_{22}=0.8\end{array}$ & $\begin{array}{l}0.00195735 \\
0.00236050 \\
0.00179132 \\
0.00219714 \\
0.00172990 \\
0.00271187 \\
0.00232818 \\
0.00210534 \\
\end{array}$ & $\begin{array}{c}0.0811064 \\
0.0020000 \\
0.0020000 \\
0.0020000 \\
49.6713000 \\
1.802000 \\
1.442000 \\
0.236148\end{array}$ & $\begin{array}{l}\text { ML } \\
\text { RO } \\
\text { ML } \\
\text { RO } \\
\text { ML } \\
\text { ML } \\
\text { ML } \\
\text { ML }\end{array}$ & $\begin{array}{l}0.00050000 \\
0.00020000 \\
0.00020000 \\
0.00533330 \\
0.00213891 \\
0.00239570 \\
0.00183331 \\
0.00226380 \\
\end{array}$ & $\begin{array}{l}0.000310016 \\
0.000000200 \\
0.000000200 \\
0.000000200 \\
0.004970960 \\
0.000187700 \\
0.000150200 \\
0.000018466 \\
\end{array}$ & $\begin{array}{l}\text { RO } \\
\text { RO } \\
\text { RO } \\
\text { RO } \\
\text { ML } \\
\text { RO } \\
\text { RO } \\
\text { RO }\end{array}$ \\
\hline 6 & $\begin{array}{l}\phi_{11}=0.8 \\
\phi_{12}=0.2 \\
\phi_{21}=-0.2 \\
\phi_{22}=0.6 \\
\theta_{11}=-0.6 \\
\theta_{12}=0.2 \\
\theta_{21}=0.2 \\
\theta_{22}=0.6\end{array}$ & $\begin{array}{l}0.00194614 \\
0.00253378 \\
0.00152255 \\
0.00222693 \\
0.00180898 \\
0.00268366 \\
0.00239610 \\
0.00216408 \\
\end{array}$ & $\begin{array}{l}0.0818381 \\
0.0020000 \\
0.0020000 \\
0.0020000 \\
1.5479300 \\
1.8020000 \\
1.4420000 \\
0.4944350 \\
\end{array}$ & $\begin{array}{l}\text { ML } \\
\text { RO } \\
\text { ML } \\
\text { RO } \\
\text { ML } \\
\text { ML } \\
\text { ML } \\
\text { ML }\end{array}$ & $\begin{array}{l}0.0005000 \\
0.0020000 \\
0.0020000 \\
0.0005333 \\
0.0017360 \\
0.0026930 \\
0.0023168 \\
0.0024620 \\
\end{array}$ & $\begin{array}{l}0.000391503 \\
0.000000200 \\
0.000000200 \\
0.000000200 \\
0.000155020 \\
0.000187700 \\
0.000150200 \\
0.000057406 \\
\end{array}$ & $\begin{array}{l}\text { RO } \\
\text { RO } \\
\text { RO } \\
\text { RO } \\
\text { RO } \\
\text { RO } \\
\text { RO } \\
\text { RO }\end{array}$ \\
\hline
\end{tabular}




\begin{tabular}{|c|c|c|c|c|c|c|c|}
\hline \multicolumn{5}{|c|}{ قبل التلويث } & \multicolumn{3}{|c|}{ بعد التلويث } \\
\hline رقم التشكيلة & طريقة التقبير القيم اضية للمعلمات & ML & Robust & Best & ML & Robust & Best \\
\hline 7 & $\begin{array}{l}\phi_{11}=0.6 \\
\phi_{12}=0.4 \\
\phi_{21}=0.3 \\
\phi_{22}=0.2 \\
\theta_{11}=0.3 \\
\theta_{12}=0.1 \\
\theta_{21}=0.2 \\
\theta_{22}=0.5\end{array}$ & $\begin{array}{l}0.00182011 \\
0.00175529 \\
0.00158952 \\
0.00156523 \\
0.00185794 \\
0.00418934 \\
0.00276094 \\
0.00196439\end{array}$ & $\begin{array}{c}0.0277795 \\
18.2857000 \\
9.33511000 \\
0.18340000 \\
19.47450000 \\
48.74230000 \\
48.74230000 \\
0.246852000\end{array}$ & $\begin{array}{l}\mathrm{ML} \\
\mathrm{ML} \\
\mathrm{ML} \\
\mathrm{ML} \\
\mathrm{ML} \\
\mathrm{ML} \\
\mathrm{ML} \\
\mathrm{ML}\end{array}$ & $\begin{array}{l}0.00022222 \\
0.00200000 \\
0.00200000 \\
0.00085714 \\
0.00168320 \\
0.00338600 \\
0.00279200 \\
0.00160400\end{array}$ & $\begin{array}{l}0.000000670 \\
0.001691860 \\
0.000947070 \\
0.000054450 \\
0.002040110 \\
0.000488670 \\
0.000488670 \\
0.000018926\end{array}$ & $\begin{array}{l}\text { RO } \\
\text { RO } \\
\text { RO } \\
\text { RO } \\
\text { ML } \\
\text { RO } \\
\text { RO } \\
\text { RO }\end{array}$ \\
\hline 8 & $\begin{aligned} \phi_{11} & =0.9 \\
\phi_{12} & =0.1 \\
\phi_{21} & =0.2 \\
\phi_{22} & =0.7 \\
\theta_{11} & =0.5 \\
\theta_{12} & =0.2 \\
\theta_{21} & =0.2 \\
\theta_{22} & =0.7\end{aligned}$ & $\begin{array}{l}0.001969780 \\
0.000704745 \\
0.001634340 \\
0.001809310 \\
0.001753060 \\
0.003484420 \\
0.003125470 \\
0.001840750\end{array}$ & $\begin{array}{c}0.0277795 \\
18.2857000 \\
9.3351100 \\
0.1834000 \\
19.4745000 \\
48.7423000 \\
48.7423000 \\
0.2468520\end{array}$ & $\begin{array}{l}\text { ML } \\
\text { ML } \\
\text { ML } \\
\text { ML } \\
\text { ML } \\
\text { ML } \\
\text { ML } \\
\text { ML }\end{array}$ & $\begin{array}{l}0.00022222 \\
0.00200000 \\
0.00200000 \\
0.00085714 \\
0.00168320 \\
0.00338600 \\
0.00279200 \\
0.00160400\end{array}$ & $\begin{array}{l}0.000000067 \\
0.001691860 \\
0.000947570 \\
0.000054450 \\
0.002040110 \\
0.000488670 \\
0.000488670 \\
0.000018926\end{array}$ & $\begin{array}{c}\text { RO } \\
\text { RO } \\
\text { RO } \\
\text { ROM } \\
\text { L } \\
\text { RO } \\
\text { RO } \\
\text { RO }\end{array}$ \\
\hline
\end{tabular}




\begin{tabular}{|c|c|c|c|c|c|c|c|}
\hline \multicolumn{5}{|c|}{ قبل التلويث } & \multicolumn{3}{|c|}{ بعد التلويث } \\
\hline رقم التشكيلة & طريقة التققير القفر اضية للمعلمات & ML & Robust & Best & ML & Robust & Best \\
\hline 9 & $\begin{array}{l}\phi_{11}=0.6 \\
\phi_{12}=0.4 \\
\phi_{21}=-0.3 \\
\phi_{22}=0.2 \\
\theta_{11}=-0.3 \\
\theta_{12}=0.1 \\
\theta_{21}=0.2 \\
\theta_{22}=0.5\end{array}$ & $\begin{array}{l}0.00183650 \\
0.00226427 \\
0.00226835 \\
0.00153823 \\
0.00218242 \\
0.00440359 \\
0.00271048 \\
0.00198422\end{array}$ & $\begin{array}{c}0.07494880 \\
12.14130010 \\
18.20250000 \\
2.67203000 \\
1.66585000 \\
8.36564000 \\
4.18382000 \\
34.47560000\end{array}$ & $\begin{array}{l}\text { ML } \\
\text { ML } \\
\text { ML } \\
\text { ML } \\
\text { ML } \\
\text { ML } \\
\text { ML } \\
\text { ML }\end{array}$ & $\begin{array}{l}0.00133333 \\
0.00200000 \\
0.00200000 \\
0.00800000 \\
0.00165187 \\
0.00484763 \\
0.00147781 \\
0.00249695\end{array}$ & $\begin{array}{c}0.00026086 \\
0.00193030 \\
0.00178275 \\
0.000133702 \\
0.000166585 \\
0.000836564 \\
0.000418380 \\
0.000344756\end{array}$ & $\begin{array}{l}\text { RO } \\
\text { RO } \\
\text { RO } \\
\text { RO } \\
\text { RO } \\
\text { RO } \\
\text { RO } \\
\text { RO }\end{array}$ \\
\hline 10 & $\begin{array}{l}\phi_{11}=-0.8 \\
\phi_{12}=-0.6 \\
\phi_{21}=-0.5 \\
\phi_{22}=0.4 \\
\theta_{11}=-0.2 \\
\theta_{12}=0.1 \\
\theta_{21}=-0.5 \\
\theta_{22}=0.3\end{array}$ & $\begin{array}{l}0.00216153 \\
0.00220332 \\
0.00218370 \\
0.00180604 \\
0.00223749 \\
0.00441582 \\
0.00168816 \\
0.00203284\end{array}$ & $\begin{array}{l}0.4701300 \\
1.9932900 \\
5.2971500 \\
1.0726100 \\
8.7182500 \\
1.4358000 \\
0.2895600 \\
39.608400\end{array}$ & $\begin{array}{l}\text { ML } \\
\text { ML } \\
\text { ML } \\
\text { ML } \\
\text { ML } \\
\text { ML } \\
\text { ML } \\
\text { ML }\end{array}$ & $\begin{array}{l}0.00450000 \\
0.00200000 \\
0.00200000 \\
0.00300000 \\
0.01199520 \\
0.01001520 \\
0.00759807 \\
0.00600506 \\
\end{array}$ & $\begin{array}{l}0.000572389 \\
0.000209329 \\
0.000679715 \\
0.000266666 \\
0.000870834 \\
0.000161169 \\
0.000304720 \\
0.004069800\end{array}$ & $\begin{array}{l}\text { RO } \\
\text { RO } \\
\text { RO } \\
\text { RO } \\
\text { RO } \\
\text { RO } \\
\text { RO } \\
\text { RO }\end{array}$ \\
\hline
\end{tabular}




\begin{tabular}{|c|c|c|c|c|c|c|c|}
\hline \multicolumn{5}{|c|}{ قبل التلويث } & \multicolumn{3}{|c|}{ بعد التلويث } \\
\hline رقم التشكيلة & طريقة التققيرير اضية للمعلمات & ML & Robust & Best & ML & Robust & Best \\
\hline 11 & $\begin{array}{l}\phi_{11}=-0.8 \\
\phi_{12}=0.6 \\
\phi_{21}=0.6 \\
\phi_{22}=-0.8 \\
\theta_{11}=-0.8 \\
\theta_{12}=0.6 \\
\theta_{21}=0.6 \\
\theta_{22}=-0.8\end{array}$ & $\begin{array}{l}0.00200798 \\
0.00188508 \\
0.00190857 \\
0.00209497 \\
0.00217061 \\
0.00249157 \\
0.00225630 \\
0.00209207\end{array}$ & $\begin{array}{l}0.275581 \\
0.950183 \\
1.336290 \\
6.274660 \\
0.205902 \\
1.372900 \\
1.372900 \\
8.237870\end{array}$ & $\begin{array}{l}\text { ML } \\
\text { ML } \\
\text { ML } \\
\text { ML } \\
\text { ML } \\
\text { ML } \\
\text { ML } \\
\text { ML }\end{array}$ & $\begin{array}{l}0.0045000 \\
0.0020000 \\
0.0020000 \\
0.0045000 \\
0.0021980 \\
0.0024620 \\
0.0022640 \\
0.0023465\end{array}$ & $\begin{array}{c}0.00095766 \\
0.00007830 \\
0.000143004 \\
0.000329157 \\
0.000021845 \\
0.000141910 \\
0.000144191 \\
0.000844106\end{array}$ & $\begin{array}{l}\text { RO } \\
\text { RO } \\
\text { RO } \\
\text { RO } \\
\text { RO } \\
\text { RO } \\
\text { RO } \\
\text { RO }\end{array}$ \\
\hline 12 & $\begin{array}{l}\phi_{11}=-0.8 \\
\phi_{12}=0.2 \\
\phi_{21}=0.2 \\
\phi_{22}=-0.8 \\
\theta_{11}=-0.8 \\
\theta_{12}=0.2 \\
\theta_{21}=0.2 \\
\theta_{22}=-0.8\end{array}$ & $\begin{array}{l}0.00206821 \\
0.00162002 \\
0.00164455 \\
0.00211576 \\
0.00220751 \\
0.00360058 \\
0.00293582 \\
0.00209540\end{array}$ & $\begin{array}{c}0.0507938 \\
12.0031000 \\
7.4499100 \\
0.3514030 \\
4.7931400 \\
14.4777000 \\
14.4777000 \\
0.3059830\end{array}$ & $\begin{array}{l}\text { ML } \\
\text { ML } \\
\text { ML } \\
\text { ML } \\
\text { ML } \\
\text { ML } \\
\text { ML } \\
\text { ML }\end{array}$ & $\begin{array}{l}0.0045000 \\
0.0020000 \\
0.0020000 \\
0.0045000 \\
0.0021980 \\
0.0033860 \\
0.0027920 \\
0.0023465\end{array}$ & $\begin{array}{l}0.000300210 \\
0.001050310 \\
0.000735616 \\
0.000017863 \\
0.000478334 \\
0.001067310 \\
0.001607310 \\
0.000067775\end{array}$ & $\begin{array}{l}\text { RO } \\
\text { RO } \\
\text { RO } \\
\text { RO } \\
\text { RO } \\
\text { RO } \\
\text { RO } \\
\text { RO }\end{array}$ \\
\hline
\end{tabular}


1- الصفاوي، صفاء يونس. (2005). "مقارنة بين المقدرات الاعتبادية و الحصينة

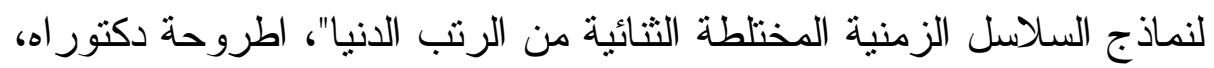
كلية علوم الحاسبات و الرياضيات، جامعة الموصل.

2- عبدالاحد، مناهل دانيال (2004). "التقدير الحصين في نموذج الانحدار الذاتي

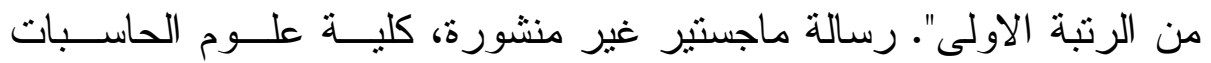

و الرياضيات، جامعة الموصل.

3- Martin ,R.D. , (1980) , “Robust Estimation of Autoregressive Models “, Indirection in Time Series, eds, D.R. Brillinger \& G.C.Tiao , Hayward , C.A. : Institute of Mathmatical Statistics .

4- Fox , A.J. , (1973)," Outliers In Time Series" , J.R. Statistics Soc. B. $34,350-363$.

5- Stochinger, N. \& Duter, R. , (1987), "Robust Time Series Analysis A survey ", Supplement To The J. Kybernetika .

6- Zch , J.E. , (1979), " Efficiency Robustness Of Generalized M-Estimates For Auto regression \& Their Use In Determining Outlier ", Ph.D. Dissertation Univ. Washington , seatle, U.S.A. 